\title{
FIT Method for Calculating Soil Particle Size Distribution from Particle Density and Settling Time Data
}

\author{
${ }^{1}$ B. KOVÁCS, ${ }^{2}$ I. CZINKOTA, ${ }^{2}$ L. TOLNER and ${ }^{3} \mathrm{GY}$. CZINKOTA \\ ${ }^{1}$ University of Szeged, Department of Mineralogy, Geochemistry and Petrology, \\ Szeged; ${ }^{2}$ Szent István University, Department of Soil Science and Agricultural \\ Chemistry, Gödöllő and ${ }^{3}$ ALTAIR Ltd., Fót (Hungary)
}

\section{Introduction}

Soil moisture and contaminant transport, erosion etc. models are widely used all over the world in the fields of geology, soil science, environmental geotechnics, contaminant hydrogeology. The mentioned models require a large amount of input data. Measurements for obtaining soil hydraulic, transport and other data are not only time-consuming, but costly as well, that is the reason why, for many applications, the prediction of these properties by pedotransfer functions (PTFs) can be a competitive alternative. In many cases the need for input parameters leads to the use of soil property databases (such as Envirobrowser of GEOREF, Inc) which is a bad alternative, as it is neither based on the behaviour of the actual soil (medium) nor on the characteristics of the permeant liquid (water, dilutant, contaminants, etc.).

Particle size distribution (PSD) is a fundamental physical property of soils, correlated to many other soil properties. As there is continuous interest in predicting more complex soil physical and chemical properties from easily measured soil characteristics it has also become a key input parameter to the PTFs (NEMES et al., 1999).

Particle size distribution (PSD) is not only a key parameter of pedotransfer functions (PTFs), but it is the basis of the petrologic classification of loose sediments (silts, sands, gravels, etc.). Despite a number of recognized international standards, soil texture data are rarely compatible across national frontiers, which make them difficult to use. Most existing PTFs adhere to the FAO/USDA system. FAO (1990) and USDA (1951) define clay as the particle size fraction $<2 \mu \mathrm{m}$, silt as the fraction between 2 and $50 \mu \mathrm{m}$ and sand as the fraction between 50 and $2000 \mu \mathrm{m}$. NEMES et al. (1999), however, gave examples for countries where soils are classified in a different manner. In most of those cases, the silt/sand boundary is defined differently - at $20 \mu \mathrm{m}$ - as adopted by the International Society of Soil Science (ISSS, 1929). Fig. 1 illustrates the used particle size classes in different particle size distribution systems.

Correspondence to: BALÁZS KOVÁCS, University of Szeged, Department of Mineralogy, Geochemistry and Petrology, H-6722 Szeged, Egyetem u. 2-6. Hungary. E-mail: kovacs.balazs@gama-geo.hu 


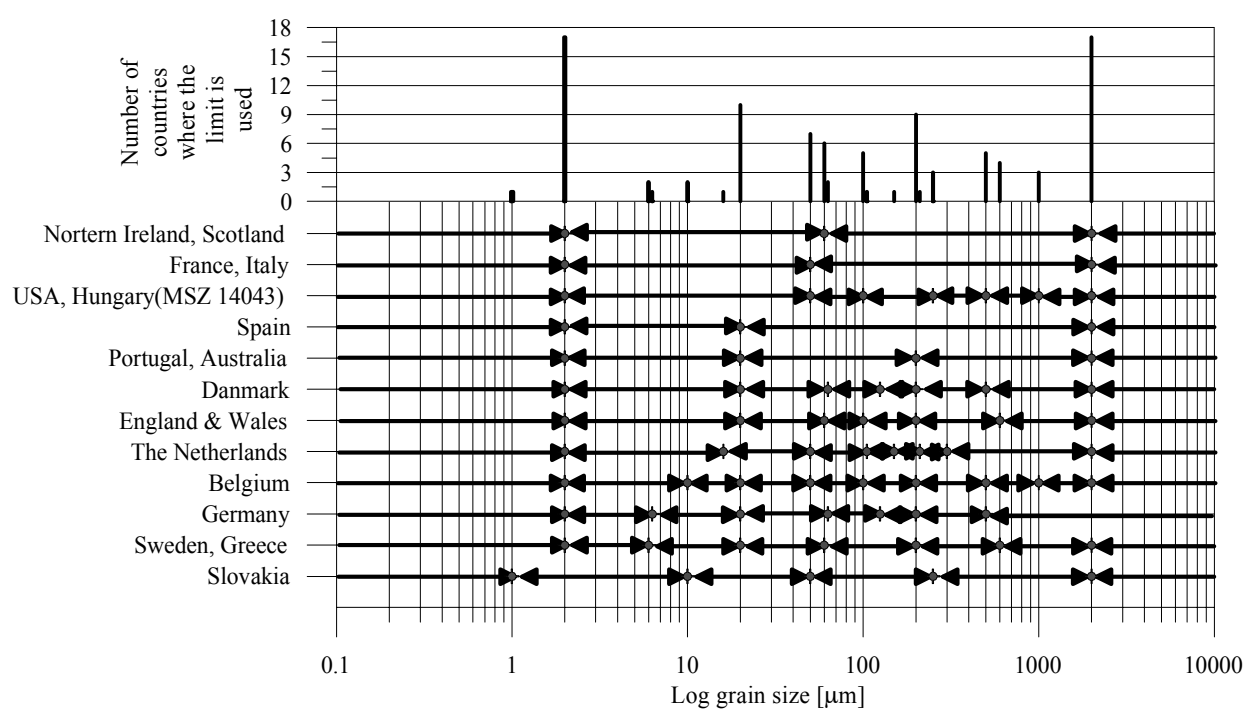

Fig. 1

The used particle size classes in different particle size distribution (PSD) systems

\section{Methods Based on Settling of Particles}

The methods based on settling particles from soil suspension use the Stokes law to determine the position of particles of different size in the suspension as a function of settling time. The Stokes law describes the settling velocity of the particles in a fluid from which the vertical settling path of the particles can be calculated during a time interval:

$$
v=\frac{2 g \cdot\left(\rho_{p}-\rho_{w}\right) \cdot r^{2}}{9 \eta},
$$

where: $v=$ the settling velocity, $\mathrm{m} \cdot \mathrm{s}^{-1} ; \rho_{p}$ and $\rho_{w}=$ the particle and fluid densities, $\mathrm{kg} \cdot \mathrm{m}^{-3} ; r=$ the radius of the particle, $\mathrm{m}$ and $\eta$ of the dynamic viscosity, Pa.s; $g=$ the acceleration of gravity, $\mathrm{m} \cdot \mathrm{s}^{-2}$.

The settling path can be achieved considering constant settling velocities from:

$$
h=\frac{2 g \cdot\left(\rho_{p}-\rho_{w}\right) \cdot r^{2}}{9 \eta} \cdot t
$$

where: $h=$ the settling path, $\mathrm{m} ; t=$ the time interval of settling, $\mathrm{s}$.

Several methods have been developed to determine the PSD curve. Most of them are based on the particle settling phenomena, but some others use different principles, like the coulter-counter or the laser beam scattering methods. (As the latter are based on different physical processes, the results obtained during the measurements are hardly comparable with the traditional methods.) The best known traditional methods are as follows: 
The pipette method. - During the test small amounts of soil suspension (samples) are taken several times from a given depth of the soil suspension settling in a cylinder with a special pipette (Andreasen- or Köhn-type pipettes). The solid content of the samples is measured by scale after drying. The particle size distribution is calculated from the weight of particles in the taken sample series.

The hydrometer method. - The hydrometer measures the average density vs. time characteristics of a soil suspension during the settling process using an aerometer (hydrometer) based on the Archimedes law. The particle size is calculated from the hydrometer geometry, the suspension density and the time using the Stokes law.

Measurement with sedimentation scale. - The sedimentation scale is a special scale with a ducked plate in the suspension, which measures the weight of the particles just settled on the plate. The PSD curve is calculated from the logged weight vs. time data series.

Methods of counter-current. - Several counter-current systems have been accomplished (STEFANOVITS et al., 1999). Some of them use air, others use different liquids with variable flow velocity against the settling of particles. The separation of particle fractions is performed by using different flow velocities; the particles having higher settling velocity than the counter-current are collected and measured.

The above-mentioned methods are widely used in the different fields of interest: agricultural sciences, geotechnics, hydrology etc. In everyday practice the hydrometer method is used by most of the institutions.

\section{Automated Soil Texture Analyzer - ASTA ${ }^{2}$ - the digital areometer}

The development of the digital areometer resulted in a stabile and linear signal generator for water level measurement. The size and energy consumption of the electronic parts of the device were reduced. This made it possible to build the electronics into the body of the aerometer. A solution for parallel measurements (using more areometers with one evaluating computer and software) has also been developed that permitted the development of the $\mathrm{ASTA}^{2}$ digital areometer by returning to the old standard hydrometer measurement principle, but introduced the continuous measurement ability and achieved much higher accuracy and linearity than earlier. The instrument head detects the change in the liquid level at the neck of the areometer, which represents the change in the density of the suspension. The equipment is shown in Fig. 2.

\section{Evaluation of Density Changes in Time Using the FIT Method}

\section{Determination of Particle Size Distribution (PSD) using FInite Tangents}

The hydrostatic approach and therefore the ASTA device generates the soil suspension density vs. time curve. The great number of measured data points led to the introduction of a new evaluation method, the Method of FInite Tangents or shortly: the "FIT Method". 


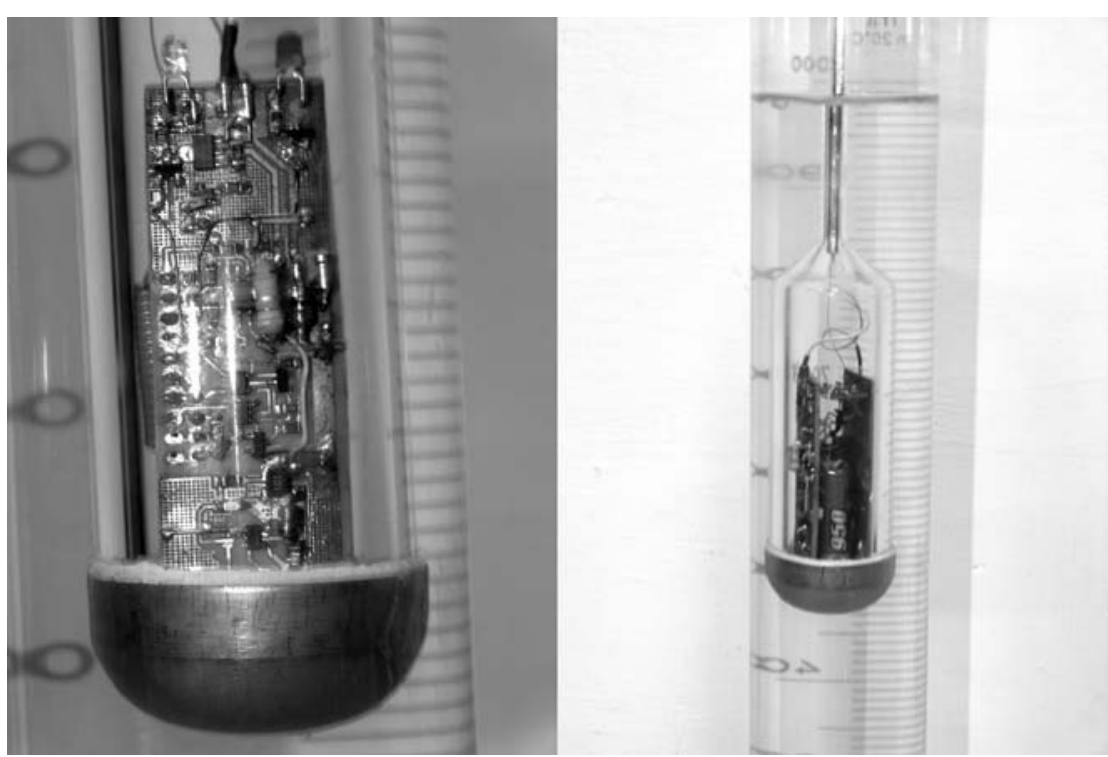

Fig. 2

Picture of the ASTA ${ }^{2}$ digital aerometer

For a better understanding of the basic idea of the FIT Method let us investigate the density changes in a mono- and a bi-disperse system. In a mono-disperse system all particles have the same density and size, while the bi-disperse system consists of a larger and a smaller grain agglomeration.

When the speed of deposition is continuous (consequence of the Stokes law), in the case of a mono-disperse system the change in density is linear, until the last particle - which is in the highest position at the beginning of the measurement merges below the reference point. After that the density is constant and equals the density of the liquid (Fig. 3, curve b, after the $t_{\underline{b}}$ point).

If the mono-disperse system is made of smaller particles, the density-time function is similar, however, the settlement of the liquid and reaching constant density need longer/more time (Fig. 3, curve a). In the case of a bi-disperse system both processes occur jointly, resulting an integrated curve of the two density-time functions (Fig. 3, curve $\mathrm{a}+\mathrm{b}$ ) (NEMES et al., 2002).

The Stokes law is only valid while the particles do not disturb each other's movement, namely the suspension is weak enough. As the whole system can be considered as the aggregation of many mono-disperse systems, it is possible to divide the measured density-time function into grain size fractions with tangent lines drawn to finite, but optional points.

The intersection point of the tangent line and the ordinate is proportional with the quantity of particles in the suspension, and the distance between the breakpoints of the tangent lines along the abscissa gives the maximum time that is needed by the particle of given size to merge below the reference point. Knowing the density and 


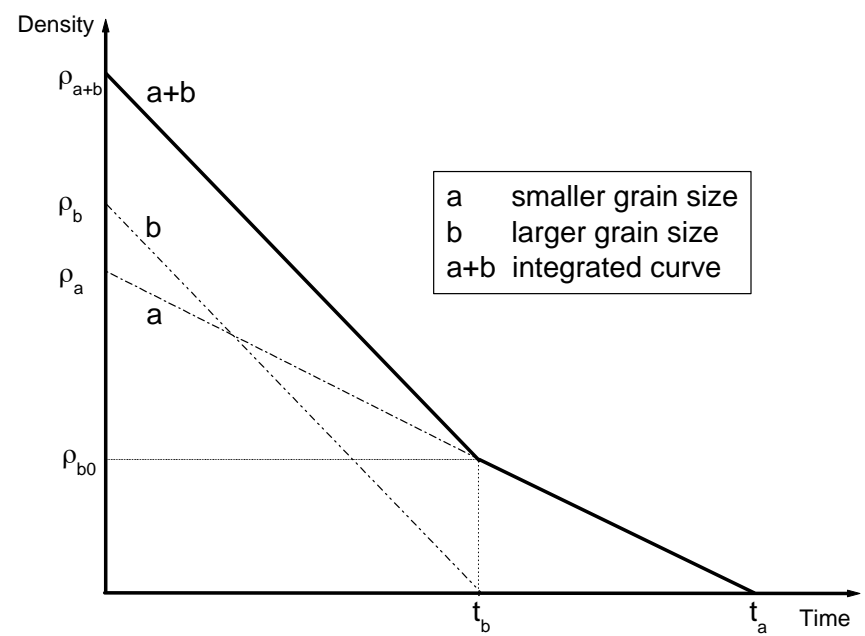

Fig. 3

Density-time characteristics of mono- ( $a, b$ curves) and bi-disperse ( $a+b$ curve) systems

the viscosity of the liquid the average particle size can be calculated using the Stokes law:

$$
r[\mu m]=10^{6} \cdot \sqrt{\frac{9 \cdot \eta[\text { Pa.s }] \cdot h[m]}{2 \cdot g\left[\frac{\mathrm{m}}{\mathrm{s}^{2}}\right] \cdot\left(\rho-\rho_{w}\right)\left[\frac{\mathrm{kg}}{\mathrm{m}^{3}}\right] \cdot t[s]}}
$$

The traditional PSD curve is obtained after normalizing the amount of substances defined by the ordinate intersections of particle size fractions.

\section{Algorithm and programming of the FIT Method}

The measured data: measurement time $t\left(t_{1}, t_{2}, \ldots t_{i}, \ldots t_{n}\right)$ and the connected density values $\rho\left(\rho_{1}, \rho_{2}, \ldots \rho_{i}, \ldots \rho_{n}\right)$. A fragmented line must be fitted on data pairs. The component line features the sum of all settling fractions at the given time. $t_{0}$ is the time when the last particle of the given fraction leaves the bottom of the areometer. In Fig. 3 , it is $t_{a}$ (the crossing of the $a$ line and t-axis) in the case of the $a$ fraction, and $\mathrm{t}_{\mathrm{b}}$ in the case of the $b$ fraction. All component lines have a known point. This is the $\rho=0, t=t_{a}$ point, in the case of the smallest fraction. While the next fraction, the sum of the last two fractions, is point $\left(\rho_{b 0}, t_{b}\right)$ in Fig. 3. $\rho_{\mathrm{b}}$ is calculable by substituting $t_{b}$ to the function of a line. For calculating the parameters of the best fitting fragmented line, a regression formula has to be derived that describes a line and crosses a given point.

The equation of a line:

$$
\rho=a \cdot t+b
$$


Substitute $\left(\rho_{0}, t_{0}\right)$ into the equation and express b:

$$
b=\rho_{0}-a \cdot t_{0}
$$

Substitute equation (5) into equation (4):

rearranged :

$$
\rho=a \cdot t-a \cdot t_{0}+\rho_{0}
$$

Transforming the variable:

$$
\rho-\rho_{0}=a \cdot\left(t-t_{0}\right)
$$

$$
y=\rho-\rho_{0} \text { and } x=t-t_{0}
$$

In further derivation $y$ means the transformed measured data and $y_{c}$ the transformed calculated data. Using transformed variables (7) the equation of the tangential line is the following:

$$
y_{c}=a \cdot x
$$

Value $a$ can be calculated as:

$$
a=\frac{\sum_{i=1}^{n}\left(y_{i} \cdot x_{i}\right)}{\sum_{i=1}^{n} x_{i}^{2}}
$$

Re-substituting the original values, by using equation (7) and (5) parameters $a$ and $b$, respectively, can be determined:

$$
\begin{aligned}
& a=\frac{\sum_{i=1}^{n}\left(\left(\rho_{i}-\rho_{0}\right) \cdot\left(t_{i}-t_{0}\right)\right)}{\sum_{i=1}^{n}\left(t_{i}-t_{0}\right)^{2}} \\
& b=\rho_{0}-a \cdot t_{0}
\end{aligned}
$$

The derived functions can be used for calculating the mass of predetermined particle size fraction.

The calculation is carried out as follows:

Step 1. Calculation of $t_{0}$ values of the predetermined fraction based on the Stokes law.

Step 2. Determination of parameters of rectangle component lines using equations (10).

Step 3. Repeating Step 2 in increasing order of each predetermined particle size fraction.

Step 4 . The cumulated particle size distribution values are the $b$ values of every component line. 


\section{Results of calculation}

Fig. 4 demonstrates the first half hour part of a real measured density signaltime curve of a volcanic soil sample originating from Tokaj (Hungary), treated with hydrogen peroxide and sodium pyrophosphate. The lines used for the calculation

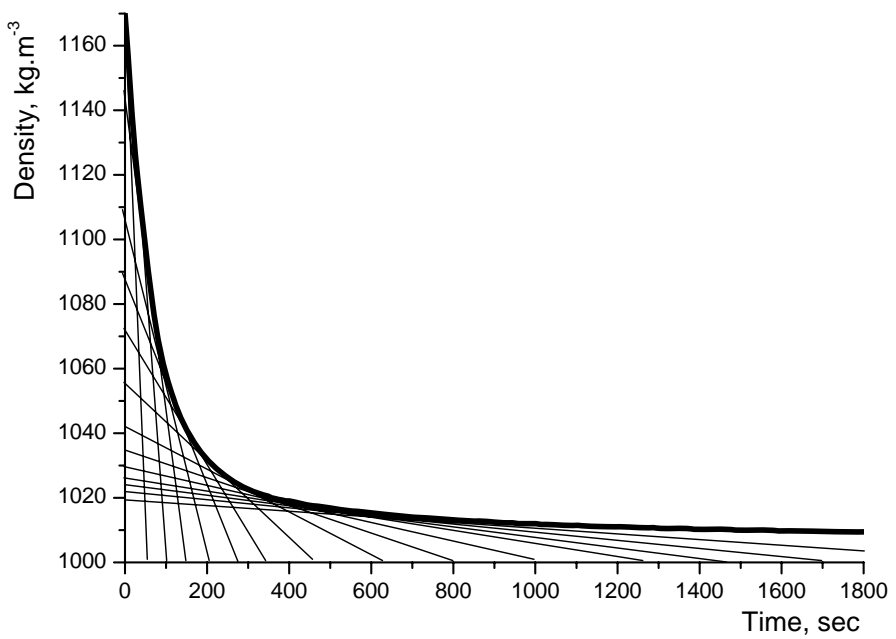

Fig. 4

The tangent lines of a measured density-time function of a volcanic soil (Tokaj, Hungary) treated with hydrogen peroxide and sodium pyrophosphate

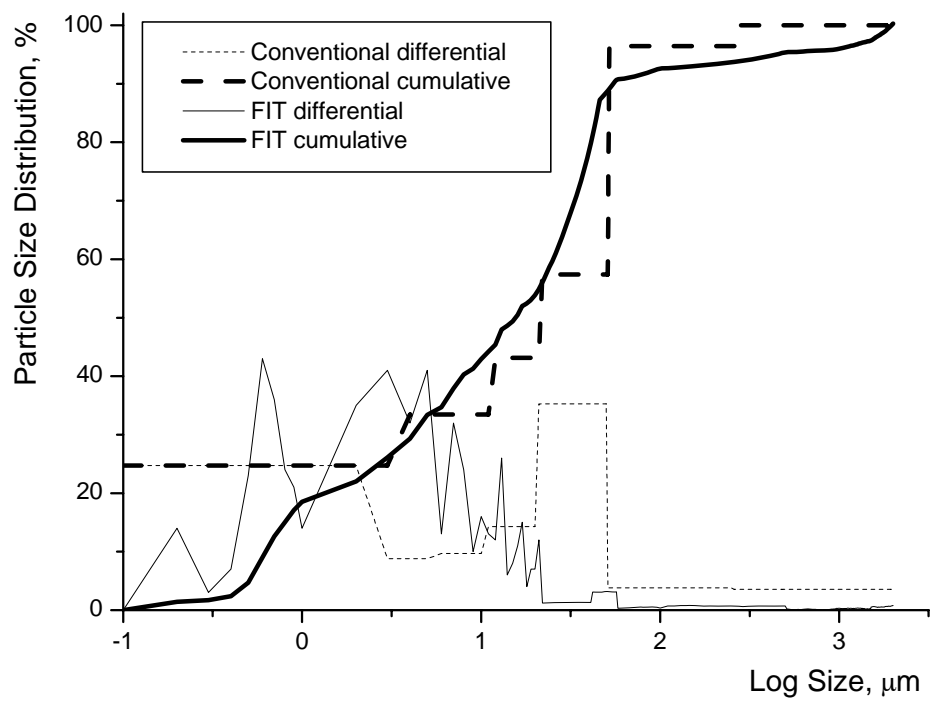

Fig. 5

Particle size distribution curves (both cumulative and differential) calculated from the tangent lines of Fig. 4 and the conventional pipette method 
(FIT Method) can also be seen in the figure. The percentage of cumulative particle size distribution is easily calculable from the cross sections of the $\mathrm{Y}$ axis, the connecting particle size can be calculated from the crossing time of tangent lines, using the Stokes law.

The cumulative and the differential particle size distribution calculated from the density signal vs. time function (Fig. 4) and results of the conventional pipette method are shown in Fig. 5. As data of the FIT calculations result in the differential curve of particle size distribution, the cumulative particle size distribution was calculated as the sum of equidistant differential results. It should be mentioned that due to the fact that the abscissa on Fig. 5 is not linear, but logarithmical, the cumulative curve in this form is not a simple sum of differential results.

\section{Conclusions}

Considering the demand for the automation of soil particle size distribution (PSD) determination the introduction of a new testing equipment was decided on. Following an overview and evaluation of the standardized methods a new calculation method was chosen to work with. The hydrostatic principle was found adequate to make automated measurements and to create high resolution PSD curves of soils. To control the theory, pilot tests were done using a recently developed prototype of the digital aerometer named Automated Soil Texture Analyzer (ASTA). The high resolution measurement results gave the opportunity to develop a new evaluation method: the method of finite tangents (FIT Method). The mentioned device and method lead to finer measurements of particle size distribution and hopefully will serve the better understanding of the environmental, geotechnical, etc. behaviour of loose sediments.

\section{Summary}

Particle size distribution (PSD) is one of the most important fundamental physical properties of soils, as it determines their physical, chemical, mechanical, geotechnical, moreover environmental behaviour. Although the measurement of PSD with different techniques is commonly performed in soil laboratories, their automation and continuous PSD curve generation have not been solved yet.

However, there are some physical principles, various sensors and different data storing methods for measuring the density-time function. In the present paper a possible solution is introduced for the measurement of the soil particle density database as a function of settling time. The equipment used for this purpose is an areometer that is widely used e.g. for determining the sugar content of must, or the alcohol content of distilled spirits, etc. The device is equipped with patent pending capacitive sensors on the neck of the areometer. It measures the changes in the water levels nearby the neck of the areometer in $1 \mu \mathrm{m}$ units with $<10 \mu \mathrm{m}$ accuracy. The typical water level changes are $3-5 \mathrm{~cm}$, which makes possible a very accurate 
determination of particle density changes due to settling in particle size analysis. The measured signals are stored in the equipment's memory and can be downloaded to the controller computer via a modified USB port. Data evaluation can be carried out online or later.

The large number of measured data points led to the introduction of a new evaluation method, the Method of FInite Tangents or shortly the "FIT Method". The dispersed soil particle system is considered as the aggregation of many monodisperse systems. From this it follows that the measured density-time function can be divided into grain size fractions with tangent lines drawn to finite, but optional points. These tangent lines are suitable for calculating the settling speed of a given fraction, as the changing speed of density is equal to the multiplication of settling speed and mass of the given grain size fraction. The settling speed of all fractions is calculable by using the Stokes law, so the mass of all of the floating fraction can be calculated. Because the soil suspension is a poly-disperse system, the measured density decrease can be considered as an integration of finite mono-disperse systems. From this, it follows that it can be interpreted as the sum of linear density vs. time functions. If the mass of each grain size fraction is known, the particle size distribution is calculable.

The method is relatively easily programmed and the intervals of grain size fractions are freely adjustable, so with this program almost all types of particle size distribution are calculable, not only those being uniform. Using the appropriate controller and evaluation program, soil particle size distribution can be calculated immediately after downloading the measured data. This technique does not need more sample preparation than past methods. The automated reading lessens the manpower required for performing the measurement - which also reduces human error sources - and provides very detailed PSD data that has advantages, among others, like revealing multi-modality in the particle-size distribution.

Key words: particle size, settling method, aerometry, calculation of SPD

The present work has been supported by the Hungarian National Scientific Research Fund (OTKA) (grants No. T 32506 and T037667) and by the Post Doctoral Research Grant of the Ministry of Education.

\section{References}

ENVIROBROWSER Environmental Constants Query Tool, 2003. GEOREF Systems Ltd. http://www.georef.com/

FoOd AND Agriculture Organisation (FAO), 1990. Guidelines For Soil Descriptions. $3^{\text {rd }}$ ed. FAO/ISRIC. Rome.

InTERNATIONAL SOCIETy OF SOIL SCIENCE, 1929. Minutes of the first Commission Meetings of the International Congress of Soil Science, Washington, 1927. In: Proceedings of the Congress of Soil Science. 4. 215-220. 
NEMES, A. et al., 1999. Evaluation of different procedures to interpolate particle-size distributions to achieve compatibility within soil databases. Geoderma. 90. 187-202.

NEMES, A. et al., 2002. Outline of an automated system for quasi-continuous measurement of particle-size distribution. Agrokémia és Talajtan. 51. 37-46.

Stefanovits, P., FileP, Gy. \& FüleKY, Gy., 1999. Soil Science. (In Hungarian) Mezőgazda Kiadó. Budapest.

United States Department of Agriculture (USDA), 1951. Soil Survey Manual. USDA Handbook No. 18. Washington, D. C. 